\title{
SÍNDROME DE GUILLAIN BARRÉ ASOCIADO A BRUCELOSIS
}

\author{
Raúl Montalvo ${ }^{1,2, a}$, Yury García ${ }^{1,2, b}$, Marcos Ñavincopa ${ }^{1,2, b}$, Eduardo Ticona $a^{1,2, b}$, \\ Gonzalo Chávez ${ }^{1,2, b}$, David A. Moore ${ }^{3, b, c}$
}

\begin{abstract}
RESUMEN
Se describe el caso de un varón de 47 años, con tiempo de enfermedad de dos días, caracterizado por pérdida de fuerza progresiva, simétrica y ascendente de miembros inferiores, se realizó punción lumbar luego de la tomografía cerebral y electromiografía lo cual evidenció polirradiculopatía motora pura con patrón axonal, compatible con el síndrome de Guillain Barré. Posteriormente, recibió cuatro sesiones de plasmaféresis, con mejoría clínica significativa desde la segunda sesión. Debido al antecedente epidemiológico se solicitó set para Brucellas, con rosa de Bengala positivo, se inició tratamiento antibiótico con rifampicina y doxiciclina, además de rehabilitación. Tres meses después el paciente mejoró completamente. La importancia del tratamiento temprano con plasmaféresis y determinar su diagnóstico etiológico hacen que el pronóstico del síndrome de Guillain Barré sea favorable.
\end{abstract}

Palabras clave: Síndrome de Guillain Barré; Brucella; Enfermedades del sistema nervioso periférico (fuente: DeCS BIREME).

\section{GUILLAIN BARRÉ SYNDROME IN ASSOCIATION WITH BRUCELLOSIS}

\begin{abstract}
We describe a case of a 47 years old male, with a history of 2 days of progressive, ascendant, symmetrical weakness in the lower extremities; a lumbar puncture was performed after the brain CT scan, as well as an electromyography, evidencing pure motor polyradiculopathy with axonal pattern, compatible with Guillain Barre syndrome. Afterwards, he received four plasmapheresis sessions, with clinical improvement from the second session. Due to his epidemiological background, Brucella set testing was done. Rose Bengal was positive, antibiotic treatment with rifampin and doxicicline was initiated, as well as rehabilitation. Three months later the patient recovered completely. The relevance of early treatment with plasmapheresis and the definition of the etiologic diagnosis determine that the prognosis of the Guillain Barre syndrome is favorable.
\end{abstract}

Key words: Guillain-Barre Syndrome, Brucella; Peripheral nervous system diseases (source: MeSH NLM).

\section{INTRODUCCIÓN}

La Brucelosis es una enfermedad infecciosa producida por cocobacilos Gram negativos aerobios inmóviles del género Brucella, tres especies son las más reconocidas como patógenos (mellitensis, abortus y suis) de las siete que se conocen a la fecha. Posee gran capacidad de sobrevivir en el interior de las células fagocíticas, lo que determina su característica clínica (1).

Las infecciones por Brucella están ocasionadas por la exposición directa o indirecta a los animales infectados (en zona urbana, a la ingesta de productos lácteos no pasteurizadas y, en zona rural, por contacto con animales enfermos). En el Perú se notifica anualmente más de 1000 casos de Brucellosis, la mayoría se deben a la infección por Brucella melitensis por consumo de queso de cabra proveniente de provincias vecinas a Lima ${ }^{(2)}$.

El periodo de incubación varía de una a tres semanas. La enfermedad puede afectar cualquier órgano o sistema y comienza con síntomas inespecíficos y polimorfos, a veces es asintomática. Generalmente se inicia con fiebre, escalofríos, malestar general, hiporexia, cefalea y artromialgias. Entre los sistemas más afectados están el tracto gastrointestinal, hepatobiliar y el sistema esquelético; en pacientes con retraso en el diagnóstico e inicio de tratamiento puede producir complicaciones focalizadas de la enfermedad.

El síndrome de Guillain Barré (SGB), es un trastorno en el que el sistema inmune lesiona en forma progresiva

\footnotetext{
Facultad de Medicina, Universidad Nacional Mayor de San Marcos. Lima, Perú.

Servicio de Enfermedades Infecciosas y Tropicales, Hospital Nacional Dos de Mayo. Lima, Perú.

Wellcome Trust Centre for Clinical Tropical Medicine, Imperial College London. London, England.

Servicio de Microbiología. Hospital Nacional Dos de Mayo. Lima, Perú.

a Médico Residente en Infectología; ${ }^{\text {b }}$ Médico infectólogo; ' Médico epidemiólogo.
}

Recibido: 07-04-10 Aprobado: 02-06-10 
la cubierta mielínica de los axones periféricos. La incidencia de compromiso neurológico por Brucelosis varía entre 1,7 a $10 \%{ }^{(3)}$. Dicho compromiso puede ser de tres formas: a) Polineuropatía inflamatoria desmielinizante aguda, que tiene como característica principal la desmielinización por parte de los macrófagos de los nervios periféricos, b) Patrón axonal, que consiste en neuropatía axonal motora aguda, c) y la forma motorsensitivo axonal. Es importante identificar los casos de polirradiculopatía ascendente debido a que sin un tratamiento precoz y apoyo ventilatorio adecuado la mortalidad es cerca al $100 \%{ }^{(4,5)}$. La finalidad de este artículo es presentar un caso de Síndrome de Guillain Barre asociado a Brucelosis.

\section{REPORTE DE CASO}

Presentamos a un paciente varón de 40 años, natural y procedente de Lima, que ingresa al Hospital Nacional Dos de Mayo con historia de dos días de enfermedad, con adormecimiento y pérdida de fuerza en extremidades inferiores de característica progresiva y simétrica, con posterior imposibilidad para desplazarse; refiere, además, disnea a esfuerzos. Al examen, se evidencia postración, lúcido y orientado en tiempo, espacio y persona, con ausencia de movimiento en miembros inferiores y tonicidad disminuida en extremidades superiores e inferiores, sensibilidad conservada, reflejos osteotendinosos ausentes. La prueba de fuerza muscular (escala 0 a 5) de las extremidades inferiores fue 0 con pruebas manuales, la cadera y los flexores plantares de ambos lados fue 1. No se detectó pérdida sensorial. Isocoria reactiva a la luz, sin signos meníngeos ni alteración de pares craneales, controla esfínteres.

El estudio de líquido cefalorraquídeo mostró xantocromía, proteinorraquia (110 $\mathrm{mg} / \mathrm{dL}$ ), pleocitosis linfocítica (50 células $/ \mathrm{mL}, 95 \%$ mononuclear) y glucosa $67 \mathrm{mg} / \mathrm{dL}$. Los exámenes de sangre de rutina y los análisis de orina no revelaron ninguna enfermedad; el ELISA para VIH y anticuerpos para Campylobacter jejuni fueron negativos.

Se realizó una electromiografía que mostró signos de polirradiculopatía motora pura con patrón axonal (latencia distal motora prolongada). El paciente inició de inmediato plasmaféresis, mostrando mejoría significativa después de la segunda sesión. La tomografía cerebral no evidenció lesiones.

Debido a que la hermana del paciente se encontraba en tratamiento para Brucelosis, se solicitó que se realice al paciente el set para Brucellas, el cual fue tomado después de la cuarta plasmaféresis, cuyos resultados son: rosa de Bengala positivo, aglutinación en placa: 1/200; 2-mercaptoetanol: 1/100; aglutinación en tubo: 1/100; prueba de flujo Lateral IgM: positivo, prueba de flujo Lateral IgG: positivo (Brucella's Kit Biomedical Research Royal Tropical Institute - Holanda).

Con estos datos se inició tratamiento antibiótico con doxiciclina $200 \mathrm{mg} /$ día y rifampicina $600 \mathrm{mg} /$ día, por 120 días. Después de tres meses de rehabilitación y terapia médica, el paciente fue capaz de desplazarse con un mínimo de asistencia y sin ningún tipo de dispositivos. Los músculos extensores, flexores y plantares han mejorado hasta grado 5 , y el resto de los músculos de las extremidades inferiores a grado 4, en forma bilateral.

\section{DISCUSIÓN}

La Brucelosis es una infección sistémica que puede comprometer cualquier órgano o sistema. Los trastornos del sistema nervioso son complicaciones poco frecuentes ${ }^{(6)}$, aunque se ha reportado casos de meningitis, abscesos, granulomas ${ }^{(7,8)}$, y espondilitis (9). Existen pocos casos publicados de síndrome de Guillain-Barré asociado a Brucelosis (Tabla 1). Uno de ellos es la publicada por Barzegar, en un niño de nueve años, que a los 10 días de historia y tratamiento para Brucelosis presenta compromiso neurológico ascendente, recibió cuatro sesiones de plasmaféresis, con resolución completa del cuadro 2 meses después (10). Otro caso reportado es la de una niña de 14 años de edad con síndrome de Guillain-Barré debido a Brucella melitensis, presentó anticuerpos séricos con títulos altos y recuperación completa después de recibir terapia adecuada (11). Kochar informó 12 casos de neurobrucelosis en India, de los cuales seis presentaron la forma de polirradiculoneuropatía proximal (12).

Se han argumentado que las complicaciones de la Brucelosis en el sistema nervioso pueden ser debido a efectos directos de citoquinas o endotoxinas presentes en los nervios periféricos, médula espinal, meninges y cerebro ${ }^{(15)}$. En el trabajo realizado por Watanabe et al. (16), demostró que los gangliósidos GM1, son moléculas expresadas por la Brucela melitensis. También señaló que la inmunización con $B$. melitensis induce la producción de anticuerpos anti-gangliósidos GM1 en ratones BALB / c. El desarrollo de este síndrome se cree que resulta del mimetismo molecular entre los gangliósidos humanos y la superficie externa bacteriana compuesto por lipooligosácaridos (17). En este sentido, los antígenos de las diferentes cepas de C. jejuni, 
Tabla 1. Casos de Brucelosis y síndrome Guillian Barré ${ }^{(10-15)}$.

\begin{tabular}{|c|c|c|c|c|c|}
\hline Publicación & $\begin{array}{c}\text { Edad } / \\
\text { sexo }\end{array}$ & $\begin{array}{l}\text { País I } \\
\text { año }\end{array}$ & Característica clínica & Diagnóstico de laboratorio & Manejo \\
\hline García ${ }^{(13)}$ & $\begin{array}{l}\text { Tres } \\
\text { casos. }\end{array}$ & $\begin{array}{l}\text { España } \\
1989\end{array}$ & $\begin{array}{l}\text { Paraparesia flácida progresiva y as- } \\
\text { cendente, hiporeflexia, parestesia. }\end{array}$ & $\begin{array}{l}\text { Prueba de Coomb. Aglutinación en } \\
\text { tubo. Electromiografía. }\end{array}$ & $\begin{array}{l}\text { Rifampicina y doxiclina. } \\
\text { Dos pacientes mejoraron después } \\
\text { de plasmaféresis, el tercero falleció. }\end{array}$ \\
\hline Al-Eissa ${ }^{(14)}$ & $\begin{array}{l}\text { Mujer, } \\
9 \text { a }\end{array}$ & $\begin{array}{l}\text { Arabia } \\
\text { Saudita } \\
1996\end{array}$ & $\begin{array}{l}\text { Hipertensión, parálisis flácida pro- } \\
\text { gresiva. }\end{array}$ & $\begin{array}{l}\text { Prueba de Coomb, Aglutinación en } \\
\text { tubo. } \\
\text { Electromiografía }\end{array}$ & $\begin{array}{l}\text { Plasmaféresis. } \\
\text { Rifampicina y doxicilina. Mejora a los } \\
2 \text { meses }\end{array}$ \\
\hline Akdeniz ${ }^{(15)}$ & $\begin{array}{l}\text { Mujer, } \\
60 \mathrm{a}\end{array}$ & $\begin{array}{l}\text { Turkia } \\
1997\end{array}$ & $\begin{array}{l}\text { Paraparesia flácida, hipotonía, hi- } \\
\text { poreflexia. }\end{array}$ & $\begin{array}{l}\text { Cultivo negativo. } \\
\text { Aglutinación en tubo, rosa de Ben- } \\
\text { gala. Electromiografía. }\end{array}$ & $\begin{array}{l}\text { Estreptomicina ( } 1,5 \text { mes) mas rifam- } \\
\text { picina y doxicilina ( } 6 \text { meses). } \\
\text { Mejora a } 1 \text { año. }\end{array}$ \\
\hline Kochar ${ }^{(12)}$ & $\begin{array}{l}\text { Varón } \\
32 \text { a }\end{array}$ & $\begin{array}{l}\text { India } \\
2000\end{array}$ & $\begin{array}{l}\text { Polirradiculopatia proximal, hipoto- } \\
\text { nia e hiporeflexia. }\end{array}$ & $\begin{array}{l}\text { ELISA, aglutinación en tubo, cultivo. } \\
\text { Electromiografía }\end{array}$ & $\begin{array}{l}\text { Plasmaféresis. } \\
\text { Estreptomicina, rifampicina y doxiclina. } \\
\text { Mejora a los } 5 \text { meses. }\end{array}$ \\
\hline Namiduru ${ }^{(11)}$ & $\begin{array}{l}\text { Mujer, } \\
14 \text { a }\end{array}$ & $\begin{array}{l}\text { Turquía } \\
2003\end{array}$ & $\begin{array}{l}\text { Parálisis flácida progresiva y simé- } \\
\text { trica ascendente. }\end{array}$ & $\begin{array}{l}\text { Hemocultivo: Brucella melitensis. } \\
\text { Anticuerpos en LCR y suero. } \\
\text { Electromiografía. }\end{array}$ & $\begin{array}{l}\text { Plasmaféresis, rifampicina y cotri- } \\
\text { moxazol ( } 4 \text { meses) } \\
\text { Mejora a los } 2 \text { meses. }\end{array}$ \\
\hline Barzegar ${ }^{(10)}$. & $\begin{array}{l}\text { Varón, } \\
9 \text { a }\end{array}$ & $\begin{array}{l}\text { Irán } \\
2009\end{array}$ & $\begin{array}{l}\text { Paraparesia y parestesia progresi- } \\
\text { va. Tiempo de enfermedad } 10 \text { días. }\end{array}$ & $\begin{array}{l}\text { Aglutinación en tubo 1/160, } \\
\text { 2-ME(negativo), electromiografía. }\end{array}$ & $\begin{array}{l}\text { Plasmaféresis, mejora a los dos me- } \\
\text { ses. No tratamiento antibiótico. }\end{array}$ \\
\hline Montalvo & $\begin{array}{l}\text { Varón, } \\
40 \text { a }\end{array}$ & $\begin{array}{l}\text { Perú } \\
2010\end{array}$ & $\begin{array}{l}\text { Pérdida de fuerza distal progresiva, } \\
\text { diagnóstico tardío de Brucellosis. }\end{array}$ & $\begin{array}{l}\text { Aglutinación en placa, Rosa de } \\
\text { Bengala positivo. Electromiografía }\end{array}$ & $\begin{array}{l}\text { Plasmaféresis, mejora a los } 3 \text { meses. } \\
\text { Doxiclina y rifampicina ( } 4 \text { meses) }\end{array}$ \\
\hline
\end{tabular}

2-ME: 2 mercaptoetanol, LCR: Líquido cefalorraquídeo.

Mycoplasma pneumoniae, Haemophilus influenzae, Brucella melitensis, Epstein-Barr, y citomegalovirus, han demostrado que imitan al GM1, GM2, GD3, GD1a, GT1a y GQ1b sugiriendo así su fuerte relación con el Síndrome de Guillain Barré ${ }^{(14,15)}$.

Nuestro paciente mostró notable mejoría clínica después de la segunda plasmaféresis (cuatro sesiones en total), y al tratamiento antibrucellósico con rifampicina y doxiciclina por cuatro meses, el cual se inicio luego de la plasmaféresis. Así mismo, antes de iniciado el cuadro de Guillain Barré, el paciente se encontraba completamente asintomático, por lo que el cuadro se habría comportado inicialmente como una Brucelosis asintomática o subclínica.

En conclusión, debe tenerse en cuenta que la polirradiculoneuropatía por Brucelosis se asemeja a una variedad de síndromes neurológicos, así mismo, en zonas endémicas como el Perú, debe considerare a la Brucelosis como causante de trastornos neurológicos. El diagnóstico etiológico de todo síndrome de Guillain Barré y del inicio terapéutico temprano con plasmaféresis, hacen que el pronóstico sea favorable.

\section{AGRADECIMIENTOS}

Al Dr. Raúl Jeri, médico neurólogo y psiquiatra consultor del Hospital Nacional Dos de Mayo por su apoyo en la evaluación neurológica del paciente.

\section{Fuente de Financiamiento}

Autofinanciado.

\section{Conflictos de Interés}

Los autores declaran no tener conflictos de interés en la elaboración ni publicación de este artículo.

\section{REFERENCIAS BIBLIOGRÁFICAS}

1. Bladwin CL, Goenka R. Host immune responses to the intracellular bacteria Brucella: does the bacteria instruct the host to facilitate chronic infection? Crit Rev Immunol. 2006; 26(5): 407-42.

2. Navarro AM, Bustamante J, Guillén A. Estrategias de prevención y control de la brucelosis humana en el Perú. Rev Peru Med Exp Salud Publica. 2005; 22(2): 87.

3. Colmenero JD, Reguera JM, Martos F, Sánchez-DeMora D, Delgado M, Causse M, Martín-Farfán A, et al. Complications associated with Brucella melitensis infection: a study of 530 cases. Medicine (Baltimore). 1996; 75(4): 195-211.

4. Cokca F, Meco O, Arasil E, Unlu A. An intramedullary dermoid cyst abscess due to Brucella abortus biotype 3 at T11-L2 spinal levels. Infection. 1994; 22(5): 359-60.

5. Bingol A, Yücemen N, Meço $O$. Medically treated intraspinal "Brucella" granuloma. Surg Neurol. 1999; 52(6): 570-76.

6. Sánchez L, Guillén A, Arreluce M, Quispe M. Brucelosis humana: estudio clínico. En: Sociedad Peruana de Medicina Interna. Libro de Resúmenes del IV Congreso de Medicina Interna. Lima: Sociedad Peruana de Medicina Interna; 1986. 
7. Ceviker N, Baykaner K, Goksel M, Sener L. Spinal cord compression due to Brucella granuloma. Infection. 1989; 17(5): 304-5.

8. Ibero I, Vela P, Pascual E. Arthritis of shoulder and spinal cord compression due to Brucella disc infection. $\mathrm{Br} \mathrm{J}$ Rheumatol. 1997; 36(3): 377-81.

9. Nas K, Gür A, Kemaloglu MS, Geyik MF, Cevik R, Büke Y, Ceviz A, et al. Management of spinal brucellosis and outcome of rehabilitation. Spinal Cord. 2001; 39(4): 22327.

10. Barzegar M, Shoaran M. Guillain Barre syndrome associated with brucellosis. Pak J Med Sci. 2009; 25(2): 329-31.

11. Namiduru M, Karaiglan I, Yilmaz M. Guillain-Barré syndrome associated with acute neurobrucellosis. Int J Clin Pract. 2003; 57(10): 919-20.

12. Kochar DK, Agarwal N, Jain N, Sharma BV, Rastogi A, Meena CB. Clinical profile of neurobrucellosis - a report on 12 cases from Bikaner (north-west India). J Assoc Physicians India. 2000; 48(4): 376-80.

13. García T, Sánchez JC, Maestre JF, Guisado F,Vilches RM, Morales B. Brucelosis y polirradiculoneuropatía inflamatoria aguda. Neurologia 1989; 4(4): 145-47
14. Al Eissa YA, Al-Herbish AS. Severe hypertension: an unusual presentation of Guillain-Barré syndrome in a child with brucellosis. Eur J Pediatr. 1996; 155(1): 53-55.

15. Akdeniz H, Irmak H, Anlar O, Demiröz AP. Central nervous system brucellosis: presentation, diagnosis and treatment. J Infect. 1998; 36(3): 297-301.

16. Shakir R, Al-Din G, Araj F, Lulu A, Mousa A. Clinical categories of neurobrucellosis. A report on 19 cases. Brain. 1987; 110(Pt 1): 213-23.

17. Watanabe K, Kim S, Nishiguchi M, Suzuki H, Watarai M. Brucella melitensis infection associated with Guillain-Barré syndrome through molecular mimicry of host structures. FEMS Immunol Med Microbiol. 2005; 45(2): 121-27.

18. Moran AP, Prendergast MM. Molecular mimicry in Campylobacter jejuni lipopolysaccharides and the development of Guillain-Barré syndrome. J Infect Dis. 1998; 178(5), 1549-50.

\section{Correspondencia: Raúl Montalvo Otivo.}

Dirección: Servicio de Enfermedades infecciosas y Tropicales, Hospital Nacional Dos de Mayo-Perú, Av. Grau s/n, Parque de la Historia de la Medicina Peruana, Lima, Perú.

Correo electrónico: otivo3@hotmail.com

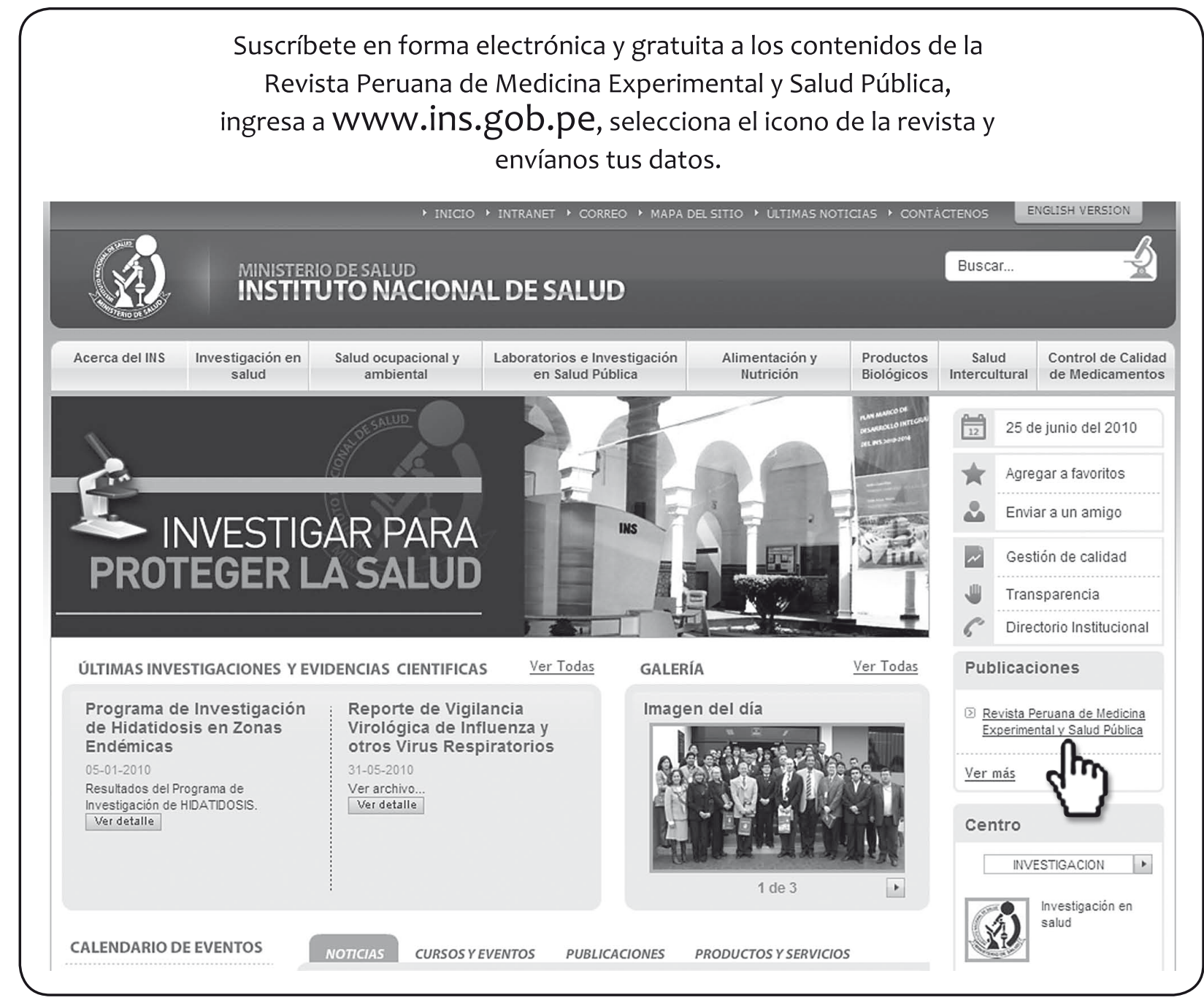

